\title{
El Instituto de Ciencias del Hombre y la Psicología española en la transición (1973-1984): una relación poco estudiada ${ }^{1}$
}

\author{
Juan A. Vera-Ferrándiz*
}

Universidad de Murcia

\begin{abstract}
Resumen El presente trabajo está destinado a subrayar la importancia histórica del Instituto de Ciencias del Hombre, una institución privada que hizo de soporte a la psicología española en un periodo muy delicado de su historia. Según tratamos de argumentar en este artículo, a partir del análisis de fuentes primarias, el Instituto pudo participar activamente en la promoción del atractivo que la psicología estaba ya despertando en un número cada vez mayor de potenciales consumidores. En efecto, en el momento en que España vivía su transición política desde la dictadura hacia la democracia, en el momento en que la propia psicología transitaba hacia su consolidación académica, científica y profesional, el Instituto de Ciencias del Hombre organizó un variado conjunto de actividades que coadyuvaron a fomentar el interés por el conocimiento psicológico. Sin embargo, y este es el centro de interés del trabajo, su labor ha pasado incomprensiblemente desapercibida para la mayor parte de los historiadores que se han dedicado al análisis institucional de la historia de la psicología en España.

Palabras clave: Instituto de Ciencias del Hombre; Historia; Psicología; España.
\end{abstract}

\section{Introducción}

El presente trabajo está consagrado a subrayar la importancia histórica de ciertas instituciones y ciertos acontecimientos que hicieron de soporte a la psicología española en el periodo de transición que supuso el paso desde su adscripción a las facultades de Filosofía y Letras, en 1968, hasta su completa independencia universitaria lograda en 1979. En este caso, concentraremos nuestra atención en el Instituto de Ciencias del Hombre (en adelante, ICH).

A diferencia de lo que ocurre con otras instituciones que participaron en el devenir de la psicología, antes de que ésta encontrase alojo en sus propias facultades, al ICH no se le ha dedicado el espacio que, en nuestra opinión, merece. En efecto, los estudios dedicados a la historia socio-institucional de la psicología en España antes de 1979 son muchos y bien documentados, y, lógicamente, se refieren a aquellas instituciones, públicas o privadas, universitarias o no, que hicieron un hueco a nuestra disciplina y posibilitaron su presencia y desarrollo: Cátedras de Psicología en las Facultades de Ciencias o de Filosofía y Letras, Junta de Ampliación de Estudios, Instituto Nacional de Psicotecnia, Departamento de Psicología Experimental del CSIC, Sociedad Española de Psicología, Revista de Psicología General y Aplicada, Colegio Oficial de Psicólogos, etc ${ }^{2}$. El caso de la Escuela Superior de Psicología y Psicotecnia de la Universidad de Madrid sería paradigmático del interés histórico que suscitan este tipo de instituciones y su importancia a la hora de reconstruir la historia de la psicología en nuestro país (véanse Bandrés y Lla-

* Dirección para correspondencia [Correspondence address]: Juan Antonio Vera Ferrándiz. Dpto. de Psicología Básica y Metodología. Campus de Espinardo. Universidad de Murcia. Aptdo. 4021. 30080 Murcia (España). E-mail: javera@um.es
Title: Instituto de Ciencias del Hombre and Spanish psychology in the transition (1973-1984): an understudied relationship.

Abstract The aim of this paper is to show the historical importance of the Instituto de Ciencias del Hombre for the development of psychology in Spain. The Instituto de Ciencias del Hombre was a private institution which gave support to Spanish psychology in a very crucial moment of its history. As identified in the analysis of primary sources, the Institute promoted the attractiveness of psychology in a growing number of potential customers. Indeed, while Spain was going through a political transition from dictatorship to democracy, and psychology was striving for its academic, scientific and professional consolidation, the Instituto de Ciencias del Hombre organized a number of activities which greatly contributed to stimulate an interest in psychological knowledge. The work of the Instituto, however, has gone inexplicably unnoticed by most of the historians devoting themselves to the institutional analysis of Spanish psychological history.

Keywords: Instituto de Ciencias del Hombre; History; Psychology; Spain.

vona, 2004 o Quintana, 2010, por solo citar dos de los más recientes estudios). Sin embargo, constituyéndose en parte fundamental del relato, de este modo no está contada toda la historia. Otras instituciones privadas también participaron en el sostén de la disciplina. Es el caso, por ejemplo, de la Fundación Juan March (Vera, 2005, 2009).

Fue estudiando el apoyo que la Fundación Juan March había prestado a la psicología española durante la década de los setenta (cf. Vera, 2010), que se descubrió la existencia del $\mathrm{ICH}$, institución que se nos antoja no menos importante que aquélla. En 1975, año en el que el ICH empezó a dar los primeros pasos para constituirse en Fundación privada, se quejaba Miguel Siguán del insuficiente apoyo institucional que recibía la psicología en España, entendiendo que "en conjunto la situación no puede considerarse como satisfactoria. Tanto el C.S.I.C., organismo gubernamental para la investigación científica, como las diferentes fundaciones privadas (Fundación March, etc.) que promueven investigaciones, hasta ahora han prestado escasa atención a la psicología.” (Siguán, 1977, p. 19). Las cosas empezaron a cambiar a partir de entonces, según tendremos ocasión de comprobar, no sólo por parte de la Juan March sino también por la participación en tal empresa de la Fundación Instituto de Ciencias del Hombre, cuya importancia socio-institucional no paró de acrecentarse durante la década de los setenta.

\footnotetext{
1 Algunos de los datos de los que aquí se informa han sido expuestos en forma de comunicación presentada al XXV Simposio de la Sociedad Española de Historia de la Psicología, Santiago de Compostela, Mayo de 2012.

${ }^{2}$ Un excelente cuadro institucional de la psicología española, extramuros de la universidad, ejecutado con la maestría puntillista de un George-Pierre Seurat, podemos encontrarlo en Quintana (2008; véase también Blanco, 1997). Para la historia de la psicología en su vertiente profesional durante los años de la transición véase Travieso, Rosa y Duro (2001).
} 
No nos cabe la menor duda de que el ICH fue importante y que muchos de sus miembros trabajaban desde y para la psicología. Sin embargo, no existen, que sepamos, estudios dirigidos a iluminar el papel que desempeñó en el escenario intelectual de aquel excitante período histórico por el que transitaban tanto la psicología española como el propio país en su conjunto. Ciertamente, la sola enumeración de las personas que participaron en esta empresa desde su creación, subraya la vocación de excelencia y su interés por proyectarse en las más diversas áreas de especialización intelectual en todo lo que toca con las ciencias del hombre: filosofía, antropología, sociología, psicología, psiquiatría, pedagogía, lingüística, etc. Y su importancia desde luego que no pasó socialmente desapercibida.

Valgan como hechos irrefutables de que los fundadores del ICH habían logrado su objetivo de penetrar con fuerza en el tejido social de la España postfranquista los dos siguientes: 1) en 1983, sólo ocho años después de su creación, la Fundación fue propuesta como candidata al Premio Príncipe de Asturias en Ciencias Sociales; y 2) un año después, en 1984, una buena representación del ICH fue recibida en audiencia por su Majestad Don Juan Carlos I en el Palacio Real de Madrid. Los nombres de los 29 intelectuales allí congregados, cuya foto puede contemplarse en la destacada noticia publicada por el diario $A B C$ (16 de diciembre de 1984, p. 7), eliminan cualquier tentación de caer en la exageración cuando se trata de evaluar el éxito de la empresa que dichos intelectuales representaban. Y si bien es cierto que la estructura organizativa y las actividades del ICH no estaban exclusivamente orientadas a la investigación y/o la docencia en psicología, tampoco lo es menos que algo de esto hubo. Algunas de las personalidades que aparecen entre los fotografiados por el colaborador del periódico $A B C$ así lo hacen suponer: entre ellos podemos encontrar a Juan Rof Carballo y a Julián Marías, a José Manuel Rodríguez-Delgado o a los mismos José Luis Pinillos y Mariano Yela, quienes, como veremos más adelante, participaron de un modo sobresaliente en las actividades del Instituto. Todos ellos constituyen un referente inequívoco de la dimensión bio-psicoantropológica de la institución, y como tales se expresaron en muchos de los Congresos, Seminarios, Cursos y demás acciones formativas que por ella fueron organizadas.

Para poner de manifiesto la conveniencia de volver la mirada histórica hacia el $\mathrm{ICH}$, a lo largo de este trabajo iremos perfilando las distintas características que lo definen. Con la intención de subrayar su importancia y penetración en la vida cultural del momento, destacaremos unas cuantas de las actividades que promovieron. El apartado final estará dedicado a estudiar las relaciones del ICH con la psicología, evaluando su participación en la popularización de la misma y en el creciente interés que se despertó en los jóvenes como materia de su formación.

\section{El nacimiento de la "Fundación Instituto de Ciencias del Hombre"}

Respecto de la fecha en que la Fundación Instituto de Ciencias del Hombre vio la luz, parece existir cierto desconcierto. Según reza en su página de 'presentación', hospedada en la web de la institución (http://www.ich.es/ presentacion.php),

la Fundación Instituto de Ciencias del Hombre nace en 1975 siendo una institución sin ánimo de lucro, con un marcado carácter humanista, teniendo como objeto principal la promoción de toda clase de actividades y estudios relacionados con las ciencias del hombre, tales como programas formativos, seminarios, coloquios, conferencias, etc. (cursivas añadidas).

Y sigue, con la intención de enfatizar la importancia de la Fundación, enumerando la lista de algunos de los intelectuales que han formado parte de la misma desde su constitución: "entre otros, las figuras de Juan Rof Carballo, Julián Marías, Federico Mayor Zaragoza, Rafael Lapesa, José Luis Pinillos, Domingo García Sabell, Mariano Yela, José Arana, Julio Caro Baroja, Pedro Laín Entralgo, .... .” (Ibidem).

Sin embargo, ni el ICH nació en 1975, ni tampoco lo hizo, estrictamente hablando, la Fundación. Según nuestros datos, el Instituto nació en algún momento de la segunda mitad del año de 1973, presentándose en público nada menos que con la organización de un Congreso Internacional sobre "La droga, problema humano de nuestro tiempo", sobre el que incidiremos más adelante; y, según consta en el Directorio de Fundaciones (MEC, 2007), se inscribió como tal Fundación el 24 de febrero de 1976.

Tampoco es fácil aclararse con respecto al organigrama de la institución en sus inicios. Según Barraquer, el Instituto fue una creación de Juan Rof Carballo y lo presidió Federico Mayor Zaragoza: "Fue inmensamente creativo -Rof- al fundar el Instituto de Ciencias del Hombre nombrando director del mismo al activísimo y culto Mayor Zaragoza." (Barraquer, 2006, p. 85). En 1984, en el preciso instante en que los recibió el Rey en audiencia y según nos informa ABC (1984, 16 de diciembre, p. 7), Rof era efectivamente el Presidente de la Fundación, Mayor Zaragoza el Director y Arana el Secretario General. Es cierto, por tanto, que Mayor Zaragoza dirigió el ICH, pero lo hizo entre 1983 y 1987, es decir, que comenzó con dicho cargo de responsabilidad prácticamente una década después de su creación. Sólo entonces alcanzó la afirmación del neurólogo rango de enunciado históricamente verdadero.

Si seguimos los anuncios aparecidos en la prensa, difícilmente podemos aclararnos acerca del staff de la institución en sus comienzos, apareciendo unas veces Rof como presidente, otras Arana, etc. Por poner tres ejemplos, en el primer recorte de prensa del que tengamos constancia que se hace mención al nacimiento del ICH, en La Vanguardia Española, se dice lo siguiente: 
El Instituto de Ciencias del Hombre es un organismo de reciente creación que se propone el estudio de los problemas humanos, del hombre mismo, desde la perspectiva de la convergencia científica y humanista. Su presidente es el doctor don José Arana, y pertenecen al grupo, entre otros, don Pedro Laín Entralgo y don Juan Rof Carballo." (Vigil, 1973, 11 de octubre, p. 11).

Aproximadamente un año después, cuando el ICH organizó su segundo Congreso Internacional, podemos leer en la sección de cultura del $A B C$ (8 de noviembre de 1974, p. 69) lo siguiente:

El cansancio de la vida se ha convertido en un fenómeno social que mina la propia vida y la sociedad, y para buscar soluciones al problema es necesario recurrir a todas las ciencias del hombre, especialmente a la Medicina, Psicología y Sociología, ha dicho don José Arana, secretario del Instituto de las (sic) Ciencias del Hombre, en la inauguración del Congreso internacional sobre «El cansancio de la vida», que se desarrolla esta semana en Madrid.

Por último, poco después de inscribirse en el registro de fundaciones, también en $A B C$, en su sección Las caras de la noticia (1977, 14 de enero, p. 5), aparece la de José Arana: "Director del Instituto de Ciencias del Hombre, de Madrid, que pronunció una interesante conferencia sobre «Familia gratuita y gratificante», dentro del ciclo organizado por el Instituto de Ciencias de la Familia." 4

Para despejar estas incógnitas, nada mejor que referirnos a los hechos legalmente sancionados. Efectivamente, el 30 de abril de 1975 se constituyó la Fundación de facto, ante el notario José María de Prada González y mediante escritura pública número 1463. Sin embargo, de iure hemos de esperar hasta la Orden de 24 de febrero de 1976 para que se reconozca, clasifique e inscriba en el Registro correspondiente de Fundaciones, según se publicó en el BOE de 3 de abril de 1976. Por este mismo BOE quedamos informados de que los fundadores aportaron una dotación inicial de un millón de pesetas y que su Consejo de Dirección o Patronato los incluiría a todos, repartiéndose entre ellos las funciones del modo en que sigue:

1. Presidente: Don Juan Rof Carballo

2. Vicepresidente: Don Julián Marías Aguilera

3. Vocales:

1. Don Rafael Lapesa Melgar

2. Don José Luis Pinillos Díaz

3. Don Mariano Yela Granizo

4. Don Primitivo de la Quintana López

\footnotetext{
${ }^{4}$ Estos bailes de fechas y nombres son, claro está, de poca trascendencia, pero resultan muy significativos desde el punto de vista de la dificultad que entraña la investigación en curso. Somos conscientes de que la solución sería bien fácil para quien tuviera acceso al fondo documental del $\mathrm{ICH}$, única forma de aliviar muchas de las dudas que ahora nos embargan, pero, el carácter de confidencialidad al que está dicho fondo sometido, por el momento hace inviable tal alternativa (de Garay, 2011, 17 de febrero). Las cursivas aplicadas a los cargos en cada una de las tres citas anteriores han sido añadidas.
}

5. Don Pedro Laín Entralgo

6. Don Álvaro Gil Varela

4. Secretario: Don José Arana Arregui

La sede de la Fundación estará domiciliada en Madrid, en la calle Ayala, número $13^{5}$.

Como puede comprobarse, en sus orígenes como Fundación, el patronato del ICH carecía de la figura de 'Director' aunque sí contara con la de 'Vicepresidente', desempeñando las funciones de 'Presidente' y 'Secretario', respectivamente, D. Juan Rof Carballo y D. José Arana Arregui, sin duda ninguna los más implicados en la promoción y gestión de la institución.

No parece necesario insistir mucho en la calidad del capital humano con que se fundó el Instituto. Además de ser innecesario, por lo conocidos que resultan esos nombres a los lectores informados, resultaría del todo punto imposible resumir en unas cuantas páginas su excelencia académica. Sólo diremos que prácticamente la totalidad de los que formaron parte en la nómina del patronato eran miembros electos de sus respectivas Academias Científicas; en ese momento de la historia, Laín, Lapesa y Marías eran Académicos de la Real, y algunos años después se sumarían a ella Rof y Pinillos. A excepción de Rof, el resto de los académicos que acabamos de mencionar obtendrían el reconocimiento del Príncipe de Asturias en sus respectivas parcelas de especialización, y, muchos de ellos, ocupaban sillones de la Academia de Ciencias Morales y Políticas. El Secretario, D. José Arana, tal vez el menos laureado, sin duda ninguna se nos manifiesta como el alma de la institución, como el responsable último de sus actividades. Refiriéndose a él, Rof Carballo cierra uno de los congresos que después tendremos oportunidad de comentar con las siguientes palabras:

¡Puede Vd. amigo Arana estar bien satisfecho de los mil desvelos y fatigas que ha costado su organización! Espero que algún día se comente y comprenda su verdadera importancia. Y por eso vaya hacia Vd. mi querido y buen amigo, mi última y más efusiva gratitud (Rof, 1975, p. 328). claro:

Y Julián Marías, cuando hace memoria, también lo deja

Hacía años que colaboraba con el Instituto de Ciencias del Hombre, de finalidad meramente intelectual, pero lo presidía mi buen amigo Juan Rof Carballo, llevaba su gestión más directa José Arana, y yo había aceptado, sin gran deseo la vicepresidencia (Marías, 2008, p. 681).

Pero contemos algo más acerca de la historia de algunos de los miembros del patronato de la recién creada Fundación. De tal modo, con los breves apuntes del próximo apartado trataremos de subrayar la existencia de algunas importantes conexiones vitales e intelectuales que nos permitan

${ }^{5}$ Escribe Francisco Umbral: "Enfermo yo, derribado y ni siquiera arcángel, Rof me atendía en su consulta de Ayala, 13.” (Umbral, 1983, 20 de junio). Es decir, en donde se domicilió la Sede Social de la Fundación ICH. 
entrever la circunstancia desde la que se proyectaba el grupo cuando se decidieron a poner en marcha el Instituto de Ciencias del Hombre allá por los inicios de la década de los setenta.

\section{El Patronato en la antesala de la fundación}

Observados en escorzo se puede decir que, desde un punto de vista intelectual, la mayoría de los fundadores son gentes que se mantuvieron más o menos distanciadas del ideario del régimen franquista, o que evolucionaron hacia el distanciamiento desde el propio régimen por la vía del desencanto, y que guardan una estrecha relación con la fe católica y un gusto por la libertad incompatible con las estrecheces de miras de la dictadura.

A decir de José Antonio Valverde, a finales de la década de los sesenta podían distinguirse en el escenario político español hasta ocho grupos, en donde se pueden encontrar a los 'cerebros' de las corrientes políticas del momento: "son nombres que, de una forma u otra, forman parte de la 'cantera' política del país y han tenido, tienen o tendrán influencia en la marcha política de España" (Valverde, 1968, p. 30). Dichos grupos, con sensibilidades propias y que él llamaba "colaboracionistas" o de "oposición moderada", son los siguientes: Falangistas, Sindicalistas (del Vertical, claro), Monárquicos, Juanistas, Carlistas, Democracia cristiana, Democracias de izquierdas y Socialistas. Es en el grupo de adscripción "más ambigua", siempre en opinión de Valverde, el de las Democracias de izquierdas, donde habría que incluir a 'cerebros' tales como Laín, Lapesa o Marías. En opinión de Valverde, estos cerebros pertenecen a personas que "son demócratas e intelectuales de tendencia socialista", y la lista se encuentra encabezada por el catedrático J. L. López Aranguren. Ésta es una opinión que, a la altura de nuestro tiempo, se puede aceptar más, menos o nada; pero, desde un punto de vista histórico sí es suficientemente ilustrativa de cómo entonces podían verse las cosas.

Por otro lado, muchos de los que aparecen en la lista de Valverde participaron en mayor o menor medida de la metafísica cristiana de Zubiri. Como señala Ruiz Calvente, "a primeros de los años cincuenta, Aranguren fue uno de los constantes asistentes a los cursos privados de Zubiri en Madrid, junto a Laín Entralgo, Rof Carballo, Vivancos, J. Marías, etc." (Ruiz Calvente, 2009, p. 1). En gran medida, también son los mismos que estuvieron implicados en empresas socio-culturales tan señaladas como el 'Congreso por la Libertad de la Cultura', la 'Sociedad de Estudios y Publicaciones' y el 'Seminario de Estudios de Humanidades', organizaciones todas ellas muy relacionadas entre sí y con las fuentes de financiación de la Fundación Ford. A este respecto, recuerda Marías (2008, pp. 408-409):

En 1960 se pudo realizar mi ya antiguo proyecto de hacer revivir el espíritu del Instituto de Humanidades, en forma bien distinta, reducido a la investigación y las publicaciones resultantes, sin docencia. La ayuda de la Ford Foundation, promovida por Waldemar Nielsen, a la So- ciedad de Estudios y Publicaciones permitió a ésta organizar un pequeño Seminario de Estudios de Humanidades, dirigido por mí, con la colaboración inmediata de los directores de las diversas secciones: Pedro Laín Entralgo, Enrique Lafuente Ferrari, Rafael Lapesa, José Luis Aranguren y Melchor Almagro ${ }^{6}$.

Los que, por la razón que fuera, todavía no habían destacado lo suficiente políticamente como para que Valverde los incluyera en sus listas, pronto vinieron a coincidir con los del grupo de las 'Democracias de izquierdas' en diversas aventuras intelectuales. Por ejemplo, en las 'Conversaciones de Gredos'. Sigamos con Marías (ob. cit., p. 287):

Desde 1951, por mayo, nos reuníamos un grupo de intelectuales (...) Los seglares más asiduos éramos Laín, Luis Rosales, Dionisio Ridruejo, Luis Felipe Vivanco, Antonio Garrigues, José luis Aranguren, José Antonio Muñoz Rojas 7 , José Corts Grau, Joaquín Ruiz Giménez, Luis Díez del Corral, José Antonio Maravall, Juan Rof Carballo; alguna vez Mariano Yela, Dámaso Alonso y otros;

El caso es que, a finales de la década de los sesenta, todos estos puntos de encuentro fueron desapareciendo. Y que, poco después, nació el Instituto de Ciencias del Hombre. Lógicamente o, mejor dicho, históricamente, no podemos más que sospechar cierta relación genética del $\mathrm{ICH}$ con esas otras organizaciones previas. Relaciones, cuya realidad y alcance, nos permiten comprender el curso seguido por el Instituto desde su misma constitución en algún momento del año de 1973.

\section{El nacimiento del ICH y su desarrollo estraté- gico}

Después de hablar del nacimiento legal de la Fundación, y de su patronato original, tenemos ahora que ocuparnos del nacimiento real del Instituto, ya que, cuando aquélla se instituía legalmente como tal Fundación, entre 1975 y 1976, éste no sólo estaba vivo sino que era muy visible en la prensa de la época y hasta disponía de una editorial propia, Karpos, con la que dar salida a su producción intelectual. A la luz de las actividades que desarrolló el Instituto durante estos primeros años de vida, trataremos de ilustrar la capacidad mostrada

${ }^{6}$ Hoy es sabido (y a Marías, cuando escribió sus memorias en los años 19871988, también debía de constarle) que las fuentes de financiación de dichas organizaciones provenían de la 'Central Intelligence Agency' americana. Entrar en detalles al respecto nos apartaría en exceso del objetivo del presente artículo, pero basta con consultar los trabajos de Glondys (s/f), Saunders (2001) y Santisteban (s/f) para que el lector pueda formarse una imagen bastante detallada de la situación.

${ }^{7}$ Sigue Marías contando que, en Gredos, hizo buena amistad con José Antonio Muñoz-Rojas, figura destacada del Banco Urquijo y director de la Sociedad de Estudios y Publicaciones. Precisamente la vía de entrada de la Fundación Ford en España: "[Nielsen] Pensó que la Ford Foundation, que no había hecho nunca nada en España, podría decidirse a iniciar alguna ayuda. Tenía que ser algo institucional, no personal. Se me ocurrió que la Sociedad de Estudios y publicaciones podría recibir un grant para organizar un Seminario de Estudios de Humanidades." (Marías, ob. cit., p. 385) 
por su patronato para ir sellando un conjunto de alianzas estratégicas que le permitieron penetrar con fuerza en la sociedad y alcanzar un notable grado de notoriedad.

En efecto, según avanzábamos más arriba, la fecha del nacimiento del ICH hay que buscarla, cuando menos, un par de años antes de que se concretara como Fundación. Así lo pone de manifiesto el hecho de que uno de sus socios fundadores, el filósofo Julián Marías, se encontrara en noviembre de 1973 impartiendo un seminario en tal institución, según informaba el diario $A B C$ a sus lectores ${ }^{8}$; o que otro de los fundadores, el lingüista Rafael Lapesa, hubiera impartido un curso universitario allí entre 1973 y 1974, según se puede leer en la contraportada de su libro 'Comunicación y Lenguaje’ (Lapesa, 1976). Por otro lado, José Arana, en la presentación de uno de los cursos universitarios organizado por el ICH en 1975, sobre el que nos detendremos más adelante, también nos pone sobre la pista al señalar que Pinillos, el ponente presentado, fue uno de los que participó en la puesta en marcha del Instituto "hace un par de años" (Arana, 1975, 30 de mayo). De igual modo, por La Vanguardia Española (Vigil, 1973, 11 de octubre) nos informamos de que los hombres del Instituto pretendían estrenarse en otoño de 1973 nada menos que con un Congreso Internacional sobre La droga, problema bumano de nuestro tiempo ${ }^{9}$. Como era de esperar, también el diario $A B C$ se haría eco de las diversas actividades que programaba el ICH desde sus comienzos, promocionándolas y siguiendo su desarrollo ${ }^{10}$. De hecho, a tres días del inicio del Congreso sobre "La droga ...", en el suplemento dominical de 21 de octubre dedicaron la portada y un reportaje de ocho páginas al fenómeno de las drogas y al tratamiento que se le iba a dispensar en la reunión organizada por el Instituto (Rof, 1973, 21 de octubre).

En fin, todo esto en 1973, es decir, cuando menos un año y medio antes de que naciera el ICH como Fundación. Para el año siguiente todavía tenían que organizar su $2^{\circ}$ Congreso Internacional, además de publicar en Seminarios y Ediciones, en su colección 'Hora $\mathrm{H}$ ', el libro que recogía las conferencias pronunciadas en este 1er. Congreso (VV.AA., 1974) $)^{11}$.

El segundo de los Congresos Internacionales que organizó el ICH tuvo como objeto El cansancio de la vida y se ce-

\footnotetext{
8 "Sigue celebrándose en el Instituto de Ciencias del Hombre el Seminario que celebra el filósofo Julián Marías sobre el tema "Aspectos de la estructura empírica de la vida"'" ( $A B C, 1973,23$ de noviembre, p. 59)

${ }^{9} \mathrm{Si}$ caemos en la cuenta de que el problema de la droga estaba siendo un referente notable en las preocupaciones de la OMS desde el inicio de la década, hasta el punto de que, en mayo de 1973, El Correo de la UNESCO dedicó su publicación mensual al 'Rompecabezas de la Droga', entenderemos que la elección del tema por parte del $\mathrm{ICH}$ no era en absoluto desatinada. ${ }^{10}$ Las relaciones entre el rotativo y el colaborador habitual del mismo, es decir, el presidente real de la Fundación, Juan Rof Carballo, hacen innecesarias cualesquiera otras hipótesis al hecho de la notable presencia de las actividades del ICH en el rotativo.

${ }^{11}$ Sobre la editorial en cuestión, Marías recuerda: "En un piso de la calle de San Lucas teníamos reuniones casi clandestinas, y en una fantasmagórica editorial, Seminarios y Ediciones, que le costó buenos dineros a Fernando Chueca, publicamos algunos libros, a fondo perdido y sin la menor esperan-
} za económica" (Marías, 2008, p. 522). lebró en la Casa Sindical de Madrid, los días 6, 7 y 8 de noviembre de 1974. Ya por La Vanguardia Española del 29 de septiembre de ese mismo año conocíamos incluso el programa del Congreso (p. 35). Y, nuevamente, ABC amplificó la importancia del mismo con dos reportajes. Uno, publicado en el mismo diario el día de su inicio y en el que se resaltaba el remoto origen de los fundadores del $\mathrm{ICH}$ :

Los creadores del Instituto -los nietos de la generación del 98- están muy ilusionados y esperanzados con su labor -ellos han tomado la antorcha casi apagada del histórico humanismo hispánico y la están alzando- y ya piensan abrir las puertas del centro para todos aquellos que deseen asociarse al instituto (de Pablos Coello, 1974, 6 de noviembre, p. 112; resaltado añadido).

$\mathrm{Y}$, el siguiente, en el semanario cultural Blanco y Negro a final de mes, en donde se realiza una larga entrevista a Rof Carballo (de la Yglesia, 1974, 30 de noviembre). Por supuesto que las colaboraciones de los participantes vieron la luz en forma de libro al año siguiente (VV.AA., 1975). Este ya sí publicado por la recién creada editorial Karpos, perteneciente al propio ICH.

Nos detendremos, finalmente, en el tercero de los congresos internacionales organizado por el $\mathrm{ICH}$, por lo que tiene de ilustrativo respecto de la política de alianzas de la institución. Nos referimos a La familia, diálogo recuperable: "es el título del próximo Congreso Internacional que el Instituto de Ciencias del Hombre anuncia para los días 3, 4 y 5 de noviembre" ( $A B C, 1975,7$ de junio, p. 11). La repercusión del congreso no se hizo esperar. Y la publicación por parte de Karpos de las diversas intervenciones, en el libro de idéntico título al año siguiente (VV.AA., 1976), fue presentada ante la prensa con la participación de importantes personalidades del momento.

En efecto, el 20 de mayo de 1976, se presentó el libro que reunía las participaciones en el congreso de noviembre del año anterior. Fue en la antigua Facultad de Medicina de San Carlos, de Madrid, donde el Ministro de Gobernación y vicepresidente del Gobierno para Asuntos del Interior, D. Manuel Fraga Iribarne, ponderaba muy positivamente la labor del ICH y daba las gracias a todas las instituciones que habían hecho posible el congreso. En la mesa se encontraban, junto al Ministro, varios de los fundadores del Instituto. "El acto terminó con unas palabras de agradecimiento del profesor Rof Carballo, otro de los autores del libro, y que ocupó un puesto en la presidencia, junto a Gabriel Cisneros y Primitivo de la Quintana. A estos últimos se les sumaron en los saludos a Fraga, Luis González Seara, Gabriel Elorriaga, el gobernador civil de Madrid y otras personas" (Beaumont, 1976, 21 de mayo, s/p). En esos momentos, el ICH ya había obtenido su reconocimiento como Fundación.

A partir de entonces, entre 1976 y 1984, la popularidad del Instituto se hizo sentir en las más variadas facetas de la vida pública, participando en la organización de diversas actividades formativas, como seminarios, conferencias, simposia, congresos nacionales e internacionales, cursos universita- 
rios e, incluso, diplomaturas para licenciados. No obstante, mientras los hombres del ICH ejercían su labor divulgadora y de organización de eventos intelectuales, en ningún momento descuidaron tampoco trabajar en el tejido de una tupida maya de relaciones institucionales, fundamentalmente con diversos ministerios. En este sentido, el Congreso de 1975 nos brinda un ejemplo esclarecedor de la capacidad de acierto del ICH, dedicando sus esfuerzos al tratamiento de temáticas muy candentes y presentes en las agendas de los responsables políticos. De tal modo, su interés por los problemas relativos a la familia se vio muy pronto recompensado, estrechándose los lazos entre la Fundación ICH y el Instituto Superior de Asesores Familiares (ISAF).

Dediquemos unas cuantas líneas más a desarrollar este argumento, analizando el contexto en el que se programó el III Congreso Internacional organizado por el ICH en 1975 y siguiendo el desarrollo posterior de las relaciones con otras instituciones. Para empezar, es digno de notar el hecho de que la noticia sobre este congreso previsto para noviembre de 1975, que como hemos visto publicaba $A B C$ el 7 de junio de ese año, aparecía inmersa en un contexto informativo más amplio. La noticia aparecía como formando parte de un anuncio en el que se daba cuenta de las resoluciones alcanzadas en el Pleno de "Política familiar en la doctrina del movimiento", en el marco del tercer Congreso Nacional de la Familia Española. Entre otras cosas, el Pleno insistía "en la necesidad de una ley Orgánica de la Familia, en la potenciación de la Delegación Nacional de la Familia y en que la protección por el Estado sea revisada y actualizada periódicamente." ( $A B C, 1975,7$ de junio, p. 11).

Es digno de notar, decíamos, por la conexión que se estableció entre los intereses temáticos del ICH y los del tercer Congreso Nacional de la Familia Española. De hecho, como respondiendo al llamamiento del Pleno, el 16 de diciembre de ese mismo año de 1975 nació el "Instituto de Ciencias de la Familia" y, por Real Decreto de 1 de septiembre de 1978, se establecieron legalmente los Servicios de Orientación Familiar (publicado en BOE de 25 de septiembre). Para cuando esto ocurría, algunos miembros del ICH, como José Arana y Mariano Yela, ya habían participando en algunas de las actividades programadas por el Instituto de Ciencias de la Familia, siendo el caso de que cuando, en 1979, se funda el Instituto Superior de Asesores Familiares (ISAF), José Arana ocuparía el cargo de Director del mismo y la docencia se le confiaría a la Fundación ICH.

No olvidemos que la Fundación ICH se inscribió en el registro a finales de febrero de 1976 y que había iniciado su andadura sólo tres años antes. Sin embargo, para finales de 1978 su notoriedad era tal que la Dirección General de la Juventud no tuvo ningún empacho en financiar sus actividades. La misma Carmela García Moreno, a la sazón Directora General de la Juventud y Promoción Socio-Cultural, informaba al diario $A B C$ de las subvenciones que se habían adjudicado al ICH para la formación de asesores familiares en el posgrado previsto (de Diego, 1980, 17 de junio).
Efectivamente, aprovechando el establecimiento oficial de los Servicios de Orientación Familiar, por Real Decreto 2275/1978 de 1 de septiembre de 1978, el ICH se hizo cargo de la organización y responsabilidad docente del ISAF, que había sido creado por la Subdirección General de la Familia, dependiente del Ministerio de Cultura $(A B C, 1979,14$ de septiembre). En octubre de 1979, el personal del ICH comenzó con la puesta en marcha del primero de los dos cursos que constituían el programa de formación del ISAF. Según se informaba, en grandes caracteres, en la sección de 'educación' del diario ABC (1979, 14 de septiembre, p. 19):

El primer curso está orientado, principalmente, a ciencias básicas de la familia para estudiar en profundidad las diversas dimensiones de la misma. Se comenzará con cursos de antropología y sociología general, para seguir con cursos sobre bases anatómicas y fisiológicas del comportamiento humano. Dentro del primer curso se incluyen también la psicopatología infantil y del adolescente, con tratamientos monográficos de la agresividad, de la drogadicción y otros. Las clases tendrán lugar de seis a nueve de la tarde (...) Las clases teóricas se entremezclarán con seminarios y con participación en investigaciones de campo.

En total, 36 asignaturas de especialización que, a decir del que fuera subdirector del centro, José Antonio Ríos, abarcaban "los aspectos sanitarios, socioculturales, jurídicos, psicológicos y pedagógicos de la familia" (Ríos, 1982, p. 60). Por lo visto, los cursos impartidos por el ISAF tuvieron que cerrar sus puertas en 1980 por falta de voluntad política y el alto coste de su organización (Castellanos, 1989).

En fin, valgan estos apretados apuntes para subrayar la vitalidad del ICH durante la primera década de su existencia. Cuando leemos los libros que recogen las intervenciones de los primeros congresos, observamos la altura de los ponentes invitados y nos preguntamos cómo era posible que una institución de reciente creación diera económicamente para tanto. En las presentaciones de dichos libros, realizadas siempre por el secretario del ICH, José Arana, encontramos algunas de las claves que permiten entender el origen de las subvenciones y ayudas. Es en el apartado de agradecimientos: "a la Organización Sindical que nos prestó sus locales y su servicios (sic) ... Pero también a muchos colaboradores anónimos que suplieron con su generosidad la escasez de medios económicos. A todos nuestro agradecimiento." (Arana, 1974, p. 11); o esta otra:

a la Dirección de la Organización Sindical, a los ministerios: Educación y Ciencia, Vivienda y Asuntos Exteriores; a los Departamentos culturales de las Embajadas de Francia y Gran Bretaña [y] en primer término a mi colaborador en las tareas de la Organización del Congreso, José Hervás (Arana, 1975, p. 3)

o también:

En nombre del Instituto de Ciencias del Hombre agradecemos sinceramente a los que han hecho posible este 
estudio convencidos de la importancia de la realidad familiar en cualquier sociedad: a los Ministerios de Asuntos Exteriores, de Educación y Ciencia y de Organización Sindical, a la distinguida familia Huarte. Escuetamente, igracias! (Arana, 1976, p. 2).

La maquinaria de establecer sinergias positivas con las instituciones del estado, según parece, estaba bien engrasada desde el inicio. El trabajo académico del grupo y su cada vez más directa implicación en las estructuras del poder, devendría en una clara ampliación de su influencia y en un reconocimiento social de la institución cada vez más evidente; hasta el punto de ser propuesta, según anunciábamos en la Introducción, como candidata al Príncipe de Asturias de Ciencias Sociales, en su edición de 1983: "Entre las candidaturas presentadas se encuentran la de los historiadores Miguel Artola y Diego Angulo, la del jurista Eduardo García de Enterría, ... y la de las Fundaciones del Instituto de Ciencias del Hombre y de Fomento de Estudios Sociales y de Sociología Aplicada (FOESA)" (ABC, 1983, 9 de junio, p. 52). El tribunal falló finalmente por Julio Caro Baroja (Fuente, 1983, 11 de junio, s/p). Como también señalábamos, sólo un año después el grupo fue recibido en audiencia real. Ocuparíamos mucho espacio poniendo nombre a los cerca de treinta asistentes al real evento. Pero la lista no tiene desperdicio. Incluyendo, además de al propio patronato original, al mismo Julio Caro Baroja, Mayor Zaragoza, Grande Covián, Santiago Grisolía, Francisco Ayala, y un excelentísimo etcétera. Innegable trascendencia la del ICH. Trascendencia que, a menos que se nos hayan escapado trabajos más informados, no se ha visto recompensada en la investigación de la historia institucional de nuestro país.

$\mathrm{Y}$, como añadiendo un punto de extrañeza a lo extraño del caso de que una institución tal haya pasado desapercibida para la historiografía española contemporánea, señalar el hecho de que ni los propios miembros del patronato original parecen concederle un lugar señalado en sus propias biografías. Sin duda, muchas y muy importantes fueron sus ocupaciones. Pero, visto lo visto, tampoco parece muy comprensible un silenciamiento tal en sus memorias. Señalémoslo, siquiera brevemente, antes de pasar a ocuparnos de las actividades del ICH más directamente relacionadas con la psicología. Veamos unos pocos, pero significativos ejemplos.

\section{E1 enigmático silenciamiento}

Laín Entralgo, en su monumental Descargo de conciencia, de 517 páginas en la edición que aquí se ha manejado (Laín, 1976/89), sólo menciona de pasada una vez al ICH, poniéndolo como ejemplo de esperanza por la atracción que despertaba en los jóvenes, $\mathrm{y}$ lo hace en el siguiente tono:

si es cierto que entre los jóvenes no se ven descollar divos semejantes a los que brillaron ayer ... también lo es que audiencias juveniles como las de Zubiri y Ochoa en la 'Sociedad de Estudios y Publicaciones' y como las de varios de los cursillos del 'Instituto de Ciencias del Hom- bre' ... en modo alguno existían hace cuarenta años" (Laín, op. cit., p. 482; cursiva añadida)

Es cierto que Laín examina el periodo de treinta años que va desde 1930 hasta 1960; pero no es menos cierto que su descargo de conciencia lo realiza entre junio de 1975 y enero de 1976, es decir, justo en el momento en que se está constituyendo legalmente la Fundación ICH.

Ítem más: cuando a Rof Carballo le tributan un merecido homenaje, en 1993 en la revista Anthropos, homenaje en el que participan los Laín, Pinillos, Yela, etc., sólo se menciona dos veces el Instituto y ninguna corre a cargo del propio Rof. Son las que siguen: "Dos décadas de colaboración intensa e íntima en la responsabilidad compartida de la dirección del Instituto de Ciencias del Hombre, me autorizan ..." (Arana, 1993, p. 42); y "Unicamente agradecer a la revista Anthropos, al Instituto de Ciencias del Hombre y, particularmente, a José Arana la ocasión que me han brindado ..." (Sánchez Asiaín, 1993, p. 38). El propio Rof (1993), aun señalando la importancia que para su propia concepción de la medicina tuvo el libro publicado por la editorial Karpos sobre Fronteras vivas del psicoanálisis (Rof, 1976), no dice ni una palabra acerca del ICH. Sorprende el silencio, cuando menos, habida cuenta que dicho libro es el resultado de unas conferencias que organizó el Instituto en 1975 y que la editorial es una creación también suya.

Por último, digamos que en las tan voluminosas como minuciosas memorias de Julián Marías, de las cuales ya hemos extractado algunas citas, el Instituto de Ciencias del Hombre sólo aparece mencionado tres veces: la primera, refiriendo una anécdota de su accidentado vuelo desde Ohio a Madrid, donde "a primera hora de la tarde tenía una conferencia de un ciclo organizado por el Instituto de Ciencias del Hombre" (Marías, 2008, p. 603); después, cuando comenta su decisión de aceptar, sin entusiasmo, la vicepresidencia del $\mathrm{ICH}$, en los términos en que ya ha sido citado más arriba; $y$, finalmente, recordando al Instituto con un lacónico: "tenía una actividad intensa y original" (Ibidem, p. 702).

Claro que no hemos leído toda la obra producida por estos renacentistas del siglo XX, pero de aquella que sí hemos tenido ocasión de disfrutar, parece no gozar de ninguna importancia el hecho de ser los promotores de una aventura intelectual, el ICH, de transcendencia innegable durante las décadas de los 70 y los 80 del siglo pasado ${ }^{12}$.

\footnotetext{
12 Mariano Yela, cuando esboza su autobiografía tampoco menciona al $\mathrm{ICH}$ en su reconstrucción, aunque, en el currículo final que añade después de la narración, sí destaca su pertenencia al grupo original que fundó el Instituto en 1973 y da cuenta de algunas de las conferencias que el mismo le patrocinó (cf. Yela, 1982).
} 


\section{E1 ICH y la Psicología}

No podemos extendernos mucho, ni entrar en el detalle de las diversas actividades organizadas por el $\mathrm{ICH}$ en las que los temas de interés psicológico fueron protagonistas. No obstante, mostraremos cómo estas preocupaciones psicológicas ocuparon un lugar destacado bien pronto en su agenda, proyectándose con ellas hacia su público potencial, convencidos tal vez de que el momento así parecía reclamarlo.

Como decimos, si el Instituto comenzó su existencia en la segunda mitad de 1973, se dejó notar enseguida la impronta psicológica de algunos de sus fundadores, ya que en 1975 Rof, Yela y Pinillos dictaron, por este orden, los primeros cursos universitarios relacionados con cuestiones psicológicas. A estos habría que sumar el que impartiera José María Rodríguez Delgado quien, sin ser formalmente parte del $\mathrm{ICH}$, desde el principio participó en sus actividades. Hay que recordar que Rodríguez Delgado había vuelto a España en 1972, con el atractivo proyecto de dirigir los departamentos de Fisiología de la Facultad de medicina de la UAM y del recién creado entonces Centro Nacional Ramón y Cajal. Y, aunque no había formado parte del grupo original del ICH, muy pronto se unió a ellos.

En total fueron seis los cursos para el año académico 1974/75, de los cuales cuatro de ellos, según veremos, estuvieron dedicados a cuestiones directamente relacionadas con lo psicológico. Los otros dos corrieron a cargo, el primero, de Julián Marías y, el último, de Rafael Lapesa.

La primera observación que habría que subrayar en este contexto tiene que ver con el hecho de que, en tan sólo un año (y recordemos, con un Congreso Internacional sobre la droga en su haber), el éxito del ICH ya era una realidad. Podemos afirmar lo anterior porque fueron precisamente razones de espacio las que obligaron a que los seminarios, conferencias y cursos que venían programando tuvieran que desplazarse a la nueva sede de la Fundación Juan March, inaugurada ese mismo año. Por lo visto, el creciente interés mostrado por el público hacia las actividades del ICH, obligó a sus organizadores a buscar instalaciones más amplias que pudieran acoger tan numerosa audiencia. El secretario del ICH, José Arana, parece dejarlo bien claro en la presentación del primer curso ofrecido:

Este año, en lugar de seminarios, puesto que el año pasado la asistencia de tanto público, particularmente de la juventud universitaria, hizo imposible el diálogo propio de seminario, este año lo hemos transformado más bien en cursos breves. Pero conferencias con el diálogo correspondiente, prácticamente según el estilo que seguimos el año pasado" (Arana, 1975, 31 de enero) ${ }^{13}$.

\footnotetext{
${ }^{13} \mathrm{La}$ fuente de la cita se encuentra en un podcast alojado en la página web de la Fundación Juan March. En el vínculo http://www.march.es/conferencias/ anteriores/ se puede escuchar la mayor parte de las conferencias que se han impartido allí desde 1975. Tratándose de la transcripción de una charla, se entiende la existencia de cierta redundancia o complicación en la construcción gramatical de la cita.
}

En efecto, Julián Marías inauguró el 31 de enero de 1975 la sala de conferencias de la nueva sede de la Juan March, con una intervención titulada "La juventud como instalación en el mundo histórico". Así, con sus peculiares y divertidos juegos del lenguaje, lo afirma él mismo al inicio de la charla:

Tengo que dar una palabra de gracias a la Fundación Juan March, que nos ha prestado su ayuda y este espléndido local en el que tengo el gusto de hablar a ustedes. Uds. saben que la palabra instalación aparece en el título de mi curso y realmente la Fundación Juan March estrena una espléndida instalación en este momento, que tengo el honor y la responsabilidad, según parece, de estrenar literalmente (Marías, 1975, 31 de enero).

Fue precisamente en esas nuevas instalaciones en donde la colaboración entre las Fundaciones ICH y Juan March hicieron un hueco a la psicología, en sus muy variadas expresiones, atacando sus problemas fundamentales y exponiendo sus fórmulas contemporáneas.

No es difícil imaginar unas salas de conferencias abarrotadas de jóvenes universitarios, estudiantes de psicología o no, completamente atrapados por las lecciones atendidas. Piénsese, si no, en el interés que hubo de despertar el segundo de los cursos universitarios programados por el ICH para el año académico de 1974/75, cuyo protagonista era José María Rodríguez Delgado y que llevaba por título "Control físico de la mente y creatividad humana"14.

En las cinco lecciones que conformaban el curso ${ }^{15}$, los presentes pudieron oír de boca de su autor las más fascinantes formas de controlar el cerebro humano y, por ende, su conducta. Películas y demostraciones sonoras en las que podían observar o escuchar el resultado de las investigaciones realizadas en las Islas Bermudas con monos gibones cerebralmente radioestimulados, según expresión del propio Rodríguez Delgado, la mundialmente famosa parada de la embestida del toro de lidia o las posibilidades de control mental a través de técnicas de 'biofeedback', tanto en animales como en humanos ${ }^{16}$. De igual modo, Delgado no dejó pasar la ocasión para tratar con su público los preocupaciones filosóficas y éticas que venían ocupándolo en los últimos tiempos, tal y como podemos leer en su libro Control físico de la mente. Hacia una sociedad psicocivilizada (Rodríguez Delgado, $1969 / 72)^{17}$.

${ }^{14}$ En vista del éxito de la convocatoria, el presentador, José Arana, reconoce que “... nosotros estamos encantados del interés que ha despertado este problema. Yo creo que para todos nosotros es un problema que atrae y un problema que nos asusta al mismo tiempo" (Arana, 1972, 18 de febrero)

${ }^{15}$ En el Apéndice están recogidos, por orden de programación, los cuatro cursos aquí analizados, incluyendo sus lecciones y otros datos de interés. 16 Por cierto, entre los presentes en el público se encontraba uno de sus ayudantes en las investigaciones con el toro electro-implantado: "uno de nuestros colaboradores en estos estudios, probablemente lo conocen ustedes. Está aquí. Los que sepan de toros reconocerán al Cordobés, que estuvo presente durante todas estas investigaciones." (Rodríguez Delgado, 1975, 25 de febrero). ¿Tal vez por si fallaba el dispositivo eléctrico?

${ }_{17}$ En la propia Fundación Juan March, a las seis de la tarde del 30 de marzo de 1973, ya tuvo lugar uno de los 'encuentros' que la misma organizaba acerca del tema Planificación cerebral del hombre futuro, en el que Rodriguez Del- 
El tercero de los cursos estuvo dedicado al psicoanálisis, siendo su ponente el presidente del ICH, Juan Rof Carballo. En Las fronteras vivas del psicoanálisis, Rof desgrana las venturas y desventuras del psicoanálisis en los contextos de la metaciencia, la epistemología, la estética o la sociología. Si unos pocos años atrás había publicado Biología y psicoanálisis (Rof, 1972), aquí retoma sus nociones centrales, sobre todo la de 'urdimbre' (primordial, constitutiva, afectiva, metaurdimbre, etc.), para proyectarse hacia las distintas fronteras que definen al psicoanálisis y lo hacen un saber, en su opinión, tan problemático como libertador. El tercero de los libros que publicó la editorial del ICH, Karpos, fue precisamente el conjunto de las cinco conferencias dadas por Rof entre los meses de marzo y abril de 1975 (Rof, 1975).

Le siguió al de Rof un curso dictado por el relativamente recientemente nombrado académico de la Real Academia de las Ciencias Morales y Políticas, Mariano Yela, en el que no se escatimaba en el uso de tecnicismos teóricos y metodológicos: La inteligencia verbal era su título ${ }^{18}$. El nivel de las clases, por mucho que no se tratara de un curso universitario ofrecido dentro de la universidad, no disminuyó en altura. Baste con un ejemplo de la primera de las lecciones, titulada Inteligencia y lenguaje, para hacernos cargo del asunto: el caso es que Yela termina, después de un cierre de sesión que no levanta ningún aplauso ni tampoco ninguna pregunta, tal vez por desconcierto o porque todavía no se ha asimilado lo escuchado, con un:

comprendo que hoy no se presta demasiado, tal vez, a preguntas concretas porque casi no hay más que 'o se admite esta metodología, por lo menos para ir trabajando y viendo a ver que da o no se admite'. Y si no se admite pues, en paz, no hay más que hablar. Muchas gracias y hasta el próximo día ${ }^{19}$.

En efecto, vectores, factores y rotaciones, coeficientes de correlación, no tuvieron que hacer muy fácil seguir alguna de las lecciones. Pero, junto a los problemas metodológicos, se ofrecía una amplia exposición de los nombres y teorías que por entonces gozaban de una alta penetración en la psicología del lenguaje (y general). Su examen incluía las más diversas fórmulas de comprensión de las relaciones entre la inteligencia y el lenguaje: el interés que el lenguaje despertó en la filosofía existencial, la filosofía analítica o la antropología psicoanalítica, etc., ya que todas descansan en su análisis, dice Yela, igual que el materialismo dialéctico (Segundo Sistema de Señales), la metafísica de la vida (Ortega y Zubiri) o los lingüistas von Humboldt, Saussure, Chomsky, etc.; y psicó-

gado actuaba como ponente y era contestado por un notable grupo de intelectuales, entre los que se incluía a J. L. Pinillos. 'Encuentro' en el que se pusieron de manifiesto las implicaciones éticas, religiosas, psicológicas, sociológicas, etc., a las que la postura del ponente comprometían (cf. VV.AA, 1973).

18 Por cierto, en el Boletín Informativo $\mathrm{n}^{\mathrm{o}} 40$ (julio-agosto) de la Fundación Juan March, cuando dan cuenta del curso de Yela, presentan al psicólogo como "fundador y consejero científico del Instituto de las (sic) Ciencias del Hombre" (p. 27)

${ }^{19}$ Ahora sí, aplausos. Cf. Yela (1975, 29 de abril). logos, claro, como los representantes de las tres tradiciones de investigación del problema que por entonces comenzaban a converger: 1) Psicología general del pensamiento; 2) la psicología genética de la inteligencia, y 3) la psicología diferencial y matemática de la inteligencia. La riqueza psicológica de las conferencias es tal que, a falta de mayor espacio, ahora nos conformamos con dejarla aquí enunciada.

Por último, José Luis Pinillos cerraba el ciclo de cursos universitarios programado para el curso 1974/75 con cinco lecciones en las que, el psicólogo, informaba de las reflexiones que desde hacía un tiempo venía volcando sobre el delicado tema de La conciencia humana.

Baste recordar que el de la conciencia será el objeto de su discurso de ingreso en la Real Academia de las Ciencias Morales y Políticas (Pinillos, 1983) o que, durante ese mismo año, vio la luz los Principios de Psicología (Pinillos, 1975), sin duda una de las obras más importantes del psicólogo bilbaíno, en donde el problema de la conciencia fue atacado desde las posiciones del evolucionismo y de todas aquellas tradiciones teóricas en las que el concepto de 'emergentismo' permite conservar las particularidades de la mente humana, sin renunciar a su origen animal. Tan importantes se nos muestran estas conferencias, tanto desde un punto de vista biográfico como teórico, que, como nos ocurría con el caso de Yela, más vale no tocarlas superficialmente y esperar a otro espacio para poder desarrollarlas. No obstante, acentuemos la importancia del momento para el propio Pinillos con unas pocas palabras suyas:

A lo largo de estos ocho o diez meses que han pasado desde que tuve no sé si la buena o mala idea de comprometerme con el Instituto de Ciencias del Hombre a hablar sobre la conciencia humana, como ustedes comprenderán he estado sumamente preocupado por el tema, que es comprometido si los hay. Como consecuencia de un sinnúmero de reflexiones, de lecturas, se me ha engrosado enormemente el calendario, el programa, y les voy a pedir a Uds. la venia, desde el primer momento, para comprimir las cinco lecciones que están anunciadas en el programa en las tres lecciones finales y precederlas de dos lecciones que juzgo, a la luz de mi situación actual, absolutamente necesarias para que cobren verdadero sentido los tratamientos de los problemas que ... voy a tratar... El tema que pretendía tratar hoy es el de la conciencia como realidad a recuperar por la psicología para un cabal estudio del comportamiento. (Pinillos, 1975, 20 de mayo)

La metamorfosis hacia una psicología con naturaleza de sistema, la integración de la conciencia en el cuerpo de reflexiones de la ciencia psicológica positiva, y, con ella, la apertura hacia el mundo de la historia, la sociedad y la cultura se estaba culminando con éxito en las elucubraciones teóricas de Pinillos ${ }^{20}$.

${ }^{20} \mathrm{Si}$ completamos este breve esquema histórico con el trabajo que Pinillos publicó en el Boletín de la propia Fundación Juan March, titulado Lo físico y lo mental (Pinillos, 1978), podemos afirmar que las conferencias pronunciadas 
La presentación del ponente, realizada por el Director de Servicios Culturales de la Fundación Juan March, el catedrático de Literatura Española Andrés Amorós, no deja lugar a dudas respecto de dos cuestiones. La primera, el éxito de la convocatoria, corroborada por el gran número de asistentes:

Antes de nada tengo que expresar en nombre de la Fundación nuestra satisfacción y nuestra gratitud al Instituto de las (sic) Ciencias del Hombre por el buen desarrollo de estos cursos que han sido seguidos por un amplio público, siempre con gran interés (Amorós, 1975, 20 de mayo)

La segunda, nos devuelve a una de las tesis que se desprende como corolario del presente estudio: aprovechándose de la oportunidad del momento histórico para impulsar actividades en las que lo psicológico fuera el tema fundamental, el ICH promovía redundantemente dicho interés que ya pudiera existir en el potencial alumnado universitario. De tal modo que las solicitudes de matrículas en psicología no hizo sino aumentar de un modo exponencial durante toda la década de los setenta; al final de la cual, se haría muy difícil oponerse a las aspiraciones de los psicólogos de conseguir Facultades propias y un Colegio profesional (Cf. Vera, 2005, 2009). Quienes trataban a nuestros hombres eran bien conscientes de ello:

Hoy les va a hablar a Uds. D. José Luis Pinillos que es, como les decía el día en que habló el profesor Yela, una de las figuras de la psicología española con un prestigio científico absolutamente reconocido e indudable $y$, además, una de las personas que han hecho posible, me parece a mí, la importancia creciente de los estudios psicológicos en España (...) Recuerdo como muchos y muchas de mis alumnos de Literatura Española se han introducido un poquito en la psicología gracias a algún manual del profesor Pinillos. Y no sólo los alumnos sino recuerdo un libro de hace ya, no sé, una década más o menos, una Introducción a la Psicología Contemporánea, que pienso que para muchos intelectuales españoles de muy diversas disciplinas, ha constituido, en efecto, una vía de acceso a los problemas psicológicos. (Amorós, idem)

\section{Conclusiones}

Hasta aquí, los resultados de nuestra investigación según hemos podido ir descubriendo gracias a las hemerotecas de los principales diarios de la época, los boletines oficiales del estado, la información y grabaciones proporcionadas por la Fundación Juan March o las publicaciones del ICH, fundamentalmente en su propia editorial Karpos. Mucho nos queda, sin embargo, por desvelar; muchas preguntas por contestar: ¿Cómo se gestó la idea de una tal institución? ¿Cuántos cursos impartieron en realidad? ¿Cómo se finan-

bien nos muestran a ese Pinillos en plena transformación teórica, que ha encontrado "una vía por la que transitar desde una resuelta afirmación de la condición científico positiva de la psicología a un reconocimiento de las condiciones singulares de la realidad de la persona y su vida biográfica." (Carpintero, 2012, pp. 22-23). ciaban sus actividades? ¿Qué tipo de público, y en qué número, asistía a sus clases, conferencias, seminarios, etc.? ¿Se conservan los programas, bibliografías, resúmenes, y demás materiales, que sabemos que se ofrecían a los asistentes? ¿Podríamos ajustar nuestras explicaciones, o reformular nuestras hipótesis históricas, a la luz de 'actas' o cualquier otro tipo de documentación oficial que generasen los miembros del Instituto en sus reuniones internas? En fin, sin du$\mathrm{da}$, todavía hay mucho por hacer.

Lo que ya hemos conseguido, no obstante, nos permite insistir en las ideas rectoras básicas de este artículo. Como es fácil advertir, de las nueve personas que participaron en la creación de la Fundación ICH, cinco destacan con luz propia en el devenir de la psicología en España, quedando el patronato de la Fundación así constituido por dos Catedráticos de Psicología (Pinillos y Yela), un médico internista, especializado en psicosomática y de orientación psicoanalista (Rof), un filósofo de amplia vocación psico-antropológica y que estuvo muy vinculado a importantes instituciones psicológicas españolas (Marías) y Pedro Laín, un Catedrático de medicina e historiador de la misma, comprometido hasta tal punto con la psicología española como para ser considerado por algunos el responsable último de su incorporación en la Universidad madrileña (Quintana, 2010). A los componentes originales del patronato pronto se les unió José María Rodríguez Delgado, incorporando en sus actividades culturales (y en sus publicaciones) la necesaria dimensión fisiológica a la explicación psicológica. En efecto, todos ellos, y algunos otros más, constituyen un referente inequívoco de la dimensión bio-psico-antropológica del ICH. Por tanto, nos parece del todo plausible señalar al ICH como una de las instituciones que contribuyó al acrecentamiento del interés por la psicología durante los años de su transición académica, científica y profesional. Aquellas "audiencias juveniles" que llenaban las salas en las que impartían algunos de sus cursos instituciones como el ICH, a decir de Laín (1976/89), lo que estaban escuchando eran informadas e interesantísimas disertaciones respecto de cosas que, hasta ese momento, sólo se podían estudiar en las Facultades de Filosofía y Letras, Sección Psicología.

Por todo ello extraña la prácticamente inexistente referencia al $\mathrm{ICH}$ en los trabajos que tratan asuntos relativos a la historia reciente de la psicología en España.

\section{Referencias}

ABC (1973, 8 de noviembre). Congreso internacional sobre el cansancio de la vida, p. 69. Recuperado de

http://hemeroteca.abc.es/nav/Navigate.exe/hemeroteca/madrid/abc/ 1974/11/08/069.html

$A B C$ (1973, 23 de noviembre). Vida humana y hombre, p. 59. Recuperado de http://hemeroteca.abc.es/nav/Navigate.exe/hemeroteca/madrid/ abc/1973/11/23/059.html

$A B C$ (1975, 7 de junio). "La familia, diálogo recuperable", p. 11. Recuperado de

http://hemeroteca.abc.es/nav/Navigate.exe/hemeroteca/madrid/abc/ 1975/06/07/039.html 
$A B C(1979,14$ de septiembre). Destinado a licenciados universitarios. Primer curso del Instituto Superior de Asesores Familiares, p. 19. Recuperado de

http://hemeroteca.abc.es/nav/Navigate.exe/hemeroteca/madrid/abc/197 9/09/14/031.htm

$A B C(1983,9$ de junio). Mañana se entrega el Príncipe de Asturias de Ciencias Sociales, p. 52. Recuperado de

http://hemeroteca.abc.es/nav/Navigate.exe/hemeroteca/madrid/abc/198 3/06/09/052.html

$A B C(1984,16$ de diciembre). Audiencia del Rey a los miembros del Instituto de Ciencias del Hombre, p. 7. Recuperado de

http://hemeroteca.abc.es/nav/Navigate.exe/hemeroteca/madrid/abc/198 4/12/16/007.html

Amorós, A. (1975, 20 de mayo). Presentación a José Luis Pinillos [Audio podcast]. Recuperado de http://www.march.es/conferencias/anteriores/voz.aspx?id=628\&l=1

Arana, J. (1974). Presentación. En VV.AA., La droga, problema bumano de nuestro tiempo (pp. 7-11). Madrid, España: Seminarios y ediciones.

Arana, J. (1975). Presentación. En VV.AA., El cansancio de la vida (pp. 1-3). Madrid, Eapaña: Karpos.

Arana, J. (1975, 31 de enero). Presentación a Julián Marías [Audio podcast]. Recuperado de http://www.march.es/conferencias/anteriores/voz.aspx?id=222\&l=1

Arana, J. (1975, 30 de mayo). Presentación a José Luis Pinillos [Audio podcast]. Recuperado de http://www.march.es/conferencias/anteriores/voz.aspx?id=632\&l=1

Arana, J. (1976). Presentación. En VV.AA., La familia, diálogo recuperable (pp. 1-2). Madrid, España: Karpos.

Arana, J. (1993). Mi encuentro con Rof. Anthropos, 141, $42-44$

Bandrés, J. y Llavona, R. (2004). La escuela de Psicología y Psicotecnia de la Universidad de Madrid (1954-1989). Psicothema, 16(2), 173-180.

Beaumont, J. F. (1976, 21 de mayo). "La familia legitima la sociedad, y esta, la familia”. Fraga, en el Instituto de Ciencias del Hombre. El País, s/p. Recuperado de http://elpais.com/diario/1976/05/21/sociedad/201477601_850215.html

Barraquer, L. (2006). Reflexiones sobre Pedro Laín Entralgo y Juan Rof Carballo. Neurología, 21(3), 162-165.

Blanco, F. (1997). Historia de la psicología española. Desde una perspectiva socio institucional. Madrid, España: Biblioteca Nueva.

Carpintero, H. (2012). José Luis Pinillos, entre el humanismo y la ciencia. Revista de Historia de la Psicología, 33(3), 7-36.

Castellanos, J. L. (1989). Orientación familiar. Papeles del Psicólogo, 39/40. Recupe rado de http:/ /www.papelesdelpsicologo.es/vernumero.asp?id=406

de Diego, E. (1980, 17 de junio). Entrevista con Carmela García Moreno. ABC, p. 36. Recuperado de http://hemeroteca.abc.es/nav/Navigate.exe/hemeroteca/madrid/abc/198 0/06/17/060.html

de Garay, V. (2011, 17 de febrero). Re: Re: Solicitud de información [Correo electrónico privado en donde se afirma la confidencialidad de la información conservada en el $\mathrm{ICH}$.

de la Yglesia, A. (1974, 30 de noviembre). Rof Carballo y el "cansancio vital". Blanco y Negro, pp. 36-37. Recuperado de http://hemeroteca.abc.es/nav/Navigate.exe/hemeroteca/madrid/blanco.y. negro $/ 1974 / 11 / 30 / 036 . h t m$

de Pablos Coello, J. M. (1974, 6 de noviembre). El cansancio de la vida. ABC, pp 109, 111, 113. Recuperado de http://hemeroteca.abc.es/nav/Navigate.exe/hemeroteca/madrid/abc/197 4/11/06/109.html

Fuente, C. (1983, 11 de junio). El antropólogo obtiene el premio Príncipe de Asturias de Ciencias Sociales. El Pais, s/p. Recuperado de http://elpais.com/diario/1983/06/11/cultura/424130408_850215.html

Glondys, O. (s/f). Causas y circunstancias del establecimiento del comité español del Congreso por la Libertad de la Cultura. Recuperado de http://www.ahistcon.org/docs/Santander/contenido/MESA $\% 208 \% 20 \mathrm{PD}$ F/Olga \%20Glondys.pdf

Laín, P. (1975/89). Descargo de conciencia. Madrid, España: Alianza.

Lapesa, R. (1976). Comunicación y lenguaje. Madrid, España: Karpos.

La V anguardia Española (1974, 29 de septiembre). Congreso internacional sobre el cansancio de la vida, p. 35. Recuperado de http://hemeroteca.lavanguardia.com/preview/1974/09/29/pagina35/34241221/pdf.html
Marías, J. (1975, 31 de enero). La juventud como instalación en el mundo bistórico [Audio podcast]. Recuperado de

http://www.march.es/conferencias/anteriores/voz.aspx?id=222\&l=1

Marías, J. (2008). Una vida presente. Memorias. Madrid, España: Páginas de espuma.

MEC (2007). Directorio de fundaciones. Recuperado de

http://www.oikos.org.es/noticias/otras/directorio_fundaciones_espanolas. pdf

Pinillos, J. L. (1975, 20 de mayo). Conciencia y comportamiento [Audio podcast]. Recuperado de http://www.march.es/conferencias/anteriores/voz.aspx?id=628\&l=1

Pinillos, J. L. (1975). Principios de Psicología. Madrid, España: Alianza.

Pinillos, J. L. (1978). Lo físico y lo mental. Boletín informativo de la Fundación Juan March, 71 (mayo), 3-31.

Pinillos, J. L. (1983). Las funciones de la conciencia. Madrid: Real Academia de CC. Morales y Políticas.

Quintana, J. (2010). José Germain y el proceso histórico de fundación de la 'Escuela de Psicología y Psicotecnia' de la Universidad de Madrid. Revista de Historia de la Psicología, 31(2/3), 23-40.

Ríos, J. A. (1982). Familia y orientación. Revista de educación, 270, 49-66.

Rodríguez Delgado, J. M. (1969/72). Control físico de la mente. Hacia una sociedad psicocivilizada. Madrid: Espasa-Calpe.

Rodríguez Delgado, J. M. (1975, 25 de febrero). Controles cerebrales: método, posibilidades y límites [Audio podcast]. Recuperado de http://www.march.es/conferencias/anteriores/voz.aspx?id=227\&l=1

Rof Carballo, J. (1972). Biología y Psicoanálisis. Bilbao, España: Desclée de Brouwer.

Rof Carballo, J. (1973, 21 de octubre). La droga y los jóvenes. Los domingos de $A B C$, pp. 4-7, 10-11, 14-15. Recuperado de http://hemeroteca.abc.es/nav/Navigate.exe/hemeroteca/madrid/abc/197 $3 / 10 / 21 / 129 . \mathrm{html}$

Rof Carballo, J. (1975). Fronteras vivas del psicoanálisis. Madrid, España: Karpos.

Rof Carballo, J. (1993). Autobiografía intelectual. Anthropos, 141, 27-37.

Ruiz Calvente, M. (2009). Zubiri, el maestro de Aranguren. A parte rei, 62, 1-18. Recuperado de http://serbal.pntic.mec.es/AParteRei

Sánchez Asíain, (1993). Rof Carballo y la importancia de lo entrañable. Anthropos, 141,38

Santisteban, F. (s/f). La Sociedad de Estudios y Publicaciones y Los comienzos de la inter vención de la Fundación Ford en España. Recuperado de http://www.ucm.es/info/hcontemp/Fabiola_Santisteban.pdf

Saunders, F. S. (2001). La CLA y la guerra fría cultural. Madrid, España: Debate.

Siguán, M. (1977). La Psicología en España. Anuario de Psicologia, 16(1), 3-22.

Travieso, D., Rosa, A. y Duro, J. C. (2001). Los comienzos de la institucionalización profesional de la psicología en Madrid. Papeles del psicólogo, 80 (noviembre), 14-29.

Umbral, F. (1983, 20 de junio). Rof Carballo. El país. Recuperado de http://elpais.com/diario/1983/06/20/sociedad/424908009_850215.html

Valverde, J. A. (1968, 16 de mayo). España hoy. Estos son los cerebros de las corrientes políticas. La Actualidad Española, pp. 28-33. Recuperado de http://www.march.es/ceacs/biblioteca/proyectos/linz/Descargas/downlo ad.aspx?file $=\mathrm{R}-76260 \&$ busqueda $=. p d$

Vigil, M. D. (1973, 11 de octubre). Próximo Congreso Internacional. La droga, problema humano de hoy. La Vanguardia Española, p. 11. Recuperado de http://hemeroteca.lavanguardia.com/preview/1973/10/11/pagina11/34297099/pdf.html

VV.AA. (1973). Planificación cerebral del hombre futuro (Colección Encuentros'). Madrid, España: Fundación Juan March.

VV.AA. (1974). La droga, problema bumano de nuestro tiempo. Madrid, España: Seminarios y ediciones.

VV.AA. (1975). El cansancio de la vida. Madrid, España: Karpos.

VV.AA. (1976). La familia, diálogo recuperable. Madrid, España: Karpos.

Vera, J. A. (2005). 1979. Un año para recordar en la historia de la psicología española. Revista de Historia de la Psicología, 26(4), 213-241.

Vera, J. A. (2009). 1979 y la psicología española, treinta años después. Revista de Historia de la Psicología, 30(2-3), 393-401.

Vera, J. A. (2010, Mayo). La Fundación Juan March y los inicios de la Psicología española moderna. Comunicación presentada al XXIII Simposio de la Sociedad Española de Historia de la Psicología, San Sebastián.

Yela, M. (1975, 29 de abril). Inteligencia y lenguaje [Audio podcast]. Recuperado de http://www.march.es/conferencias/anteriores/voz.aspx?id=623\&l=1

Yela, M. (1982). Mariano Yela: esbozo de autobiografía. Revista de Historia de la Psicología, 3(4), 281-332.

(Articulo recibido: 15-3-2013; revisado: 28-5-2013; aceptado: 29-5-2013) 


\section{Apéndice}

Los cuatro cursos están ordenados temporalmente según fueron dictados. En la página web de la Fundación Juan March se conserva el audio de todos ellos, excepto del segundo de Rodríguez Delgado. La columna de la derecha informa del tiempo de duración de cada conferencia. Las fotos han sido extraídas de la propia Fundación Juan March

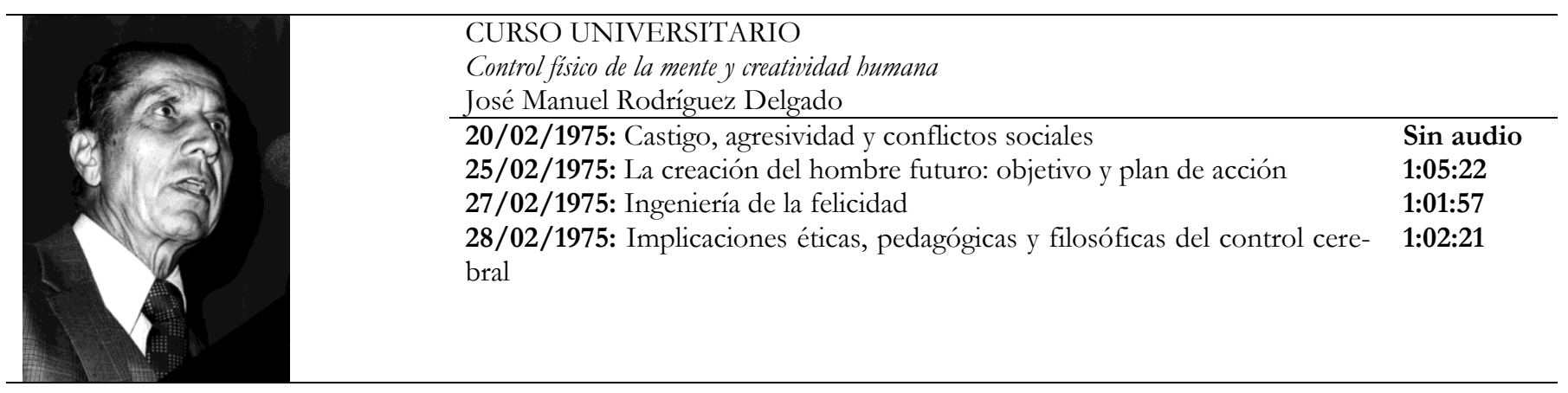

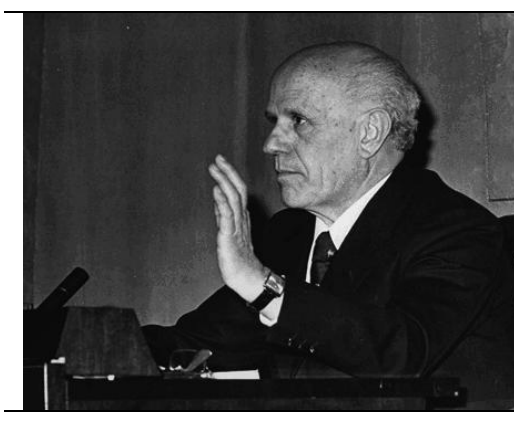

CURSO UNIVERSITARIO

Las fronteras vivas del psicoanálisis

Juan Rof Carballo

07/03/1975: Metaciencia y psicoanálisis

$0: 59: 41$

11/03/1975: La frontera epistemológica del psicoanálisis

1:04:37

14/03/1975: La frontera estética. Psicoanálisis de la creatividad 1:04:55

artística

04/04/1975: La frontera sociológica. Sociología del conocimiento 1:09:56

08/04/1975: Ciencias del hombre y 'ethos' contemporáneo 1:02:27

\begin{tabular}{l|l} 
CURSO UNIVERSITARIO \\
La inteligencia verbal \\
Mariano Yela Granizo
\end{tabular}

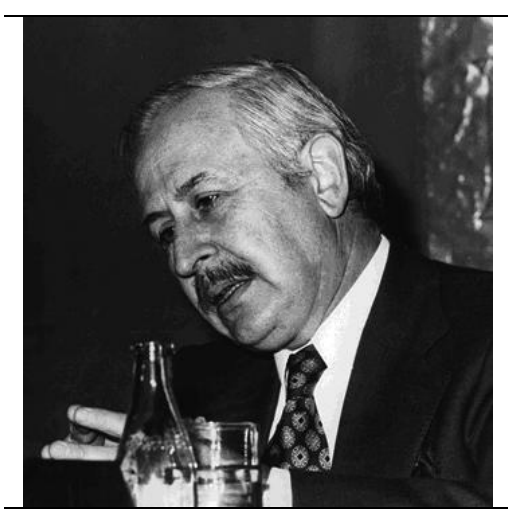

CURSO UNIVERSITARIO

La conciencia bumana

José Luis Pinillos Díaz

20/05/1975: Conciencia y comportamiento

1:22:07

23/05/1975: La conciencia como reflejo

1:16:55

26/05/1975: Origen y función biológica de la conciencia

$1: 20: 18$

27/05/1975: La conciencia sobre sí misma

1:19:30

30/05/1975: El problema del dualismo en la psicología contemporánea

1:10:09 